\title{
Clinical Efficacy of Cardiac Resynchronization Therapy in Patients with Ischemic and Non-ischemic Cardiomyopathy
}

\author{
MOHAMMED RUZIEH, MD, OSAMA DASA, MD, ASSIA MEZIANE-TANI, MD, ANAS RENNO, MD, \\ TURKI ALKULLY, MD, UMAR DARR, MD, BLAIR GRUBB, MD and LAURA MURPHY, MD
}

Division of Cardiology, Department of Medicine, The University of Toledo Medical Center, OH

\begin{abstract}
We evaluated the response of cardiac resynchronization therapy $(C R T)$ in ischemic cardiomyopathy (ICMP) and non-ischemic cardiomyopathy (NICMP). We determined if the cause of cardiomyopathy, ischemic versus non-ischemic, affects CRT outcomes in regard to New York Heart Association (NYHA) category and ejection fraction. We used a retrospective chart review for adult patients who underwent biventricular device placement between January 2005 and July 2015 at the university of Toledo medication center as part of their standard of care. NYHA category and ejection fraction were compared before and after CRT in both ICMP and NICMP patients. The NYHA category improved by $0.39 \pm 0.90$ and $0.81 \pm 0.79(p=0.001)$ in ICMP and NICMP, respectively. The NYHA category worsened on follow-up in 11 (11.2\%) patients in the ICMP group and five $(5.2 \%)$ patients in the NICMP group $(p=0.12)$. A total of $50(51 \%)$ patients in the ICMP group and 26 (26.8\%) in the NICMP had no improvement in their NYHA category $(p<0.001)$. Thirty-seven (37.8\%) patients in the ICMP group and $66(68 \%)$ in the NICMP group showed improvement by one or more in their NYHA categories $(p<0.001)$. NICMP remained a significant predictor of response to CRT independent of other variables after controlling it using logistic regression analysis. The ejection fraction increased by $5.58 \pm 11.41$ in ICMP compared with $12.86 \pm 14.55$ in NICMP $(p=0.001)$. Our study shows an improvement in NYHA functional class and ejection fraction after CRT therapy in heart failure patients regardless of etiology. However, a more favorable outcome was noticed in the NICMP subgroup. A future randomized control trial with a control group that specifically addresses this question and investigates possible etiologies is warranted.
\end{abstract}

KEYWORDS. Cardiac pacing, cardiac resynchronization therapy, heart failure, implantable defibrillation therapy.
ISSN 2156-3977 (print) ISSN 2156-3993 (online)

(C) 2016 Innovations in Cardiac Rhythm Management

\section{Introduction}

Heart failure remains one of the leading causes for cardiovascular hospitalizations and outpatient office visits.

The authors report no conflicts of interest for the published content. Manuscript received June 15, 2016, Final version accepted July 11, 2016.

Address correspondence to: Mohammed Ruzieh, MD, 3000 Arlington Ave, Toledo, OH 43614, USA.

E-mail: mohammed.ruzieh@utoledo.edu
The lifetime risk for developing heart failure in Americans above 40 years old is about $20 \%{ }^{1}$

In 2010, there were 1,023 million hospital discharges with heart failure as the primary diagnosis. ${ }^{2}$ The total cost of heart failure in 2013 was $\$ 32$ billion and it is projected to increase to $\$ 70$ billion by $2030 .^{2,3}$

Despite enhancements in pharmacologic treatment, heart failure still carries a poor prognosis. ${ }^{4}$ Left ventricular dyssynchrony resulting from interventricular conduction delay reduces the ejection fraction $(\mathrm{EF}) .^{5} \mathrm{~A}$ biventricular pacemaker is thought to resynchronize the abnormal left 
ventricular contraction without affecting the heart rate or oxygen consumption. ${ }^{5}$

Multiple clinical trials have proven that cardiac resynchronization therapy (CRT) using a biventricular pacemaker improves all-cause mortality and hospitalization in patients with low EF and wide QRS complex. ${ }^{6-9}$ Additional trial series showed improvement in exercise capacity, New York Heart Association (NYHA) class, quality of life, and EF. ${ }^{10-13}$

On the basis of the available data, the American Heart Association recommends CRT for patients with ischemic cardiomyopathy (ICMP) and non-ischemic cardiomyopathy (NICMP), NYHA class II, III, or IV, EF $\leq 35 \%$, and wide QRS with or without left bundle branch block (LBBB) on maximum medical therapy. ${ }^{14}$

However, limited data are available comparing the efficacy of CRT between ICMP and NICMP, particularly in standard clinical practice. In this study, we evaluated the improvement in NYHA and EF among patients with ICMP and NICMP who received CRT as part of their standard of care.

\section{Methodology}

The study was approved by the institutional review board. We conducted a retrospective chart review for all patients who underwent biventricular device placement between January 2005 and July 2015.

\section{Study population}

Subjects had systolic heart failure on maximally tolerated medical therapy for at least 3 months before CRT implantation.

A total of 238 patients who had CRT were identified. Baseline characteristics, medications, NYHA category, and EF were collected within 3 months before the biventricular device placement and up to 1 year after. Follow-up NYHA categories were obtained from the heart failure clinic notes. The follow-up EF was estimated using echocardiogram.

All patients had their epicardial lead placed in the lateral ventricular wall between the base and the apex.

Patients who had no follow-up in our heart failure center and had no follow-up echocardiogram available at the time of data collection were excluded from the study.

The primary outcome of the study was a change in the NYHA category in both ICMP and NICMP and EF as documented by echocardiogram before and after CRT.

\section{Statistical analysis}

Statistical analyses were carried out using the SPSS 21.0 software (SPSS Inc.)

Continuous variables were compared using the two-sample $\mathrm{t}$-test and were expressed as means \pm standard deviation. The chi-square test was used to compare baseline characteristics. Multivariate logistic regression was then applied to control for confounders and determine if other variables were significantly related to the outcome of our study (response to CRT).
A two-tailed $p<0.05$ was considered to be statistically significant.

\section{Results}

Between January 2005 and July 2015, 238 patients underwent biventricular device placement. Of these 238 patients, 43 patients did not have follow-up data available in our hospital database and were excluded from the study. Twelve had biventricular pacemaker placement without a defibrillator.

The clinical characteristics of the 195 patients included in the study are summarized in Table 1. Age, male gender, history of coronary artery bypass graft (CABG), and creatinine levels were significantly different between ICMP and NICMP $(\mathrm{p}<0.05)$.

Changes in NYHA class and EF as documented by echocardiogram are summarized in Table 2.

NYHA category improved by $0.39 \pm 0.90$ and $0.81 \pm 0.79$ $(\mathrm{p}=0.001)$ in ICMP and NICMP, respectively. NYHA category worsened on follow-up in $11(11.2 \%)$ patients in the ICMP group and five (5.2\%) patients in the NICMP group $(p=0.12)$. A total of $50(51 \%)$ patients in the ICMP group and $26(26.8 \%)$ in the NICMP had no improvement in their NYHA category $(\mathrm{p}<0.001)$. Thirty-seven $(37.8 \%)$ patients in the ICMP and $66(68 \%)$ in the NICMP showed improvement by one or more in their NYHA categories $(\mathrm{p}<0.001)$ (Figure 1).

NICMP remained a significant predictor of response to CRT independent of other variables after controlling it using logistic regression analysis.

Female gender and QRS complex $>150$ ms were both significant predictors of response to CRT when adjusted to other variables using logistic regression (odds ratio 2.44 and 2.51, respectively) (Table 3).

The EF increased by $5.58 \pm 11.41$ in ICMP compared with $12.86 \pm 14.55$ in NICMP $(\mathrm{p}=0.001)$.

\section{Discussion}

The results of this study show that cardiac resynchronization therapy improves two important markers of cardiac function and clinical status in patients with heart failure and wide QRS complex. NYHA class and EF had modest improvement in patients with ICMP, whereas patients with NICMP benefit significantly more than their ischemic counterparts.

Previous studies suggested that cardiac resynchronization produced clinical benefits in patients with heart failure and intraventricular conduction delay. ${ }^{10,12}$ In one trial, ${ }^{10} 67$ patients were evaluated but only 25 patients had heart failure of ischemic origin, which might lead to more a favorable outcome. Another randomized controlled trial of 453 patients concluded that cardiac resynchronization improves the 6-minute walk test, quality of life, and EF without significant differences between patients with ischemic or non-ischemic origin of their heart failure. In general, studies investigating outcomes of cardiac resynchronization therapy have focused on risk factors affecting morbidity and mortality without differentiating between ischemic and non-ischemic subgroups. 
Table 1: Characteristics of the patients at baseline

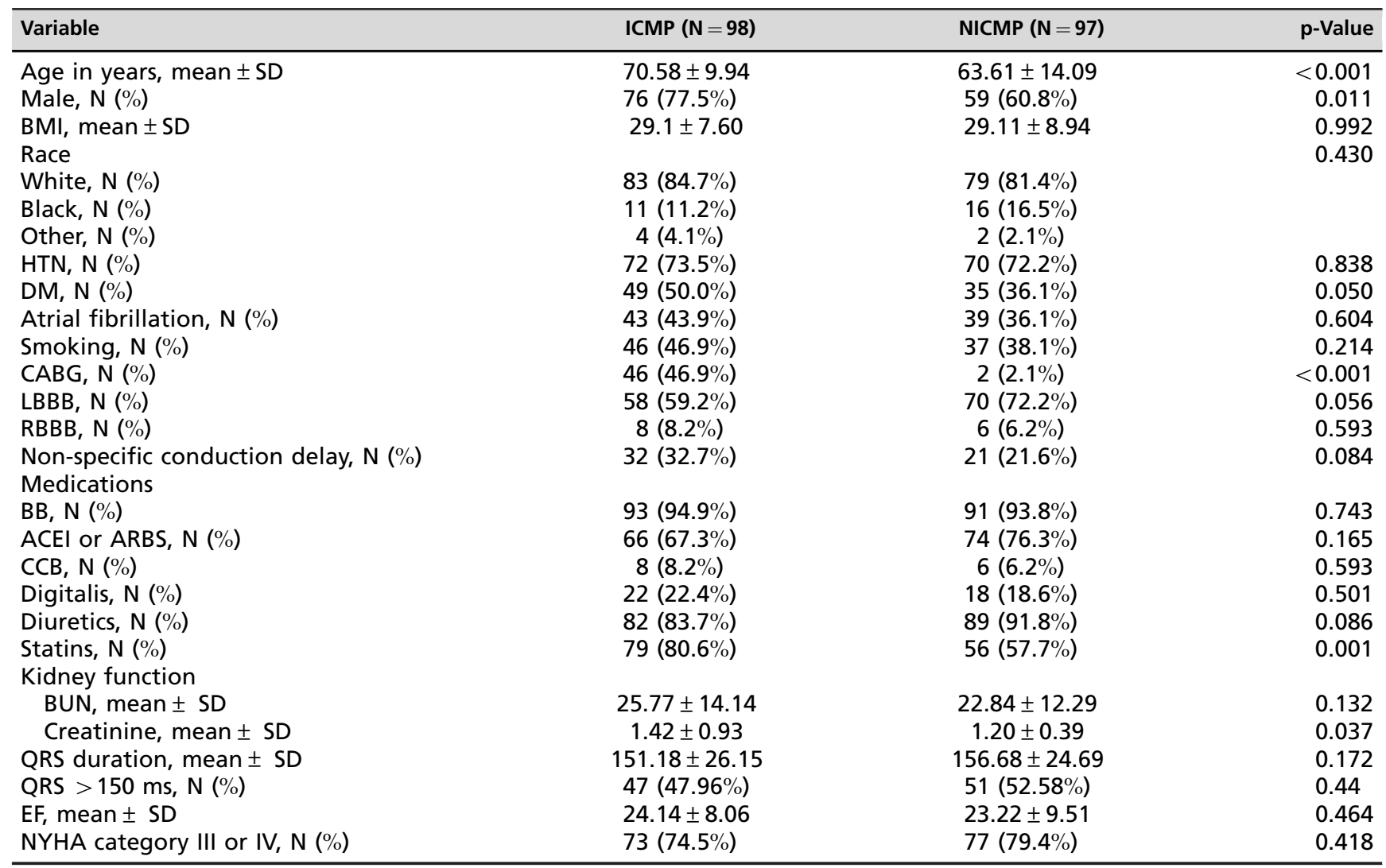

ACEI: Angiotensin Converting Enzyme Inhibitor; ARBS: Angiotensin Receptor Blockers; BB: $\beta$-blockers; BMI: body mass index; BUN: blood urea nitrogen; CABG: coronary artery bypass graft; CCB: calcium channel blockers; DM: diabetes mellitus; EF: ejection fraction; HTN: hypertension; LBBB: left bundle branch block; NYHA: New York Heart Association; SD: standard deviation.

Table 2: Outcomes of the study for all the patients in accordance with their NYHA category and EF.

\begin{tabular}{|c|c|c|c|}
\hline Variable & ICMP $(\mathrm{N}=98)$ & NICMP $(\mathbf{N}=97)$ & p-Value \\
\hline Change in NYHA, mean $\pm S D$ & $0.39 \pm 0.90$ & $0.81 \pm 0.79$ & 0.001 \\
\hline Change in $\mathrm{EF}$, mean $\pm \mathrm{SD}$ & $5.58 \pm 11.41$ & $12.86 \pm 14.55$ & 0.001 \\
\hline No change in NYHA category & Total $\mathrm{N}=50(51 \%)$. & Total $\mathrm{N}=26(26.8 \%)$ & $<0.001$ \\
\hline Baseline NYHA III & 29 & 18 & \\
\hline Baseline NYHA II & 17 & 7 & \\
\hline Rise in NYHA category by $\geq 1$ & Total $\mathrm{N}=37(37.8 \%)$ & Total $\mathrm{N}=66(68 \%)$ & $<0.001$ \\
\hline Baseline NYHA II & 3 & 11 & \\
\hline Rise in NYHA category by $\geq 2$ & Total $\mathrm{N}=11(11.2 \%)$ & Total $\mathrm{N}=18(18.6 \%)$ & 0.15 \\
\hline Baseline NYHA IV & 4 & 7 & \\
\hline Baseline NYHA III & 7 & 11 & \\
\hline
\end{tabular}

EF: ejection fraction; N: number; NYHA: New York Heart Association; SD: standard deviation.

Our study re-examines CRT into these two divided groups. We believe ischemia plays a significant role in how CRT improves a patient's cardiac function and overall lifestyle, evaluated in our review by EF and New York Heart Association classification.

Of 195 patients with CRT examined after exclusion, the EF and NYHA class were affected by whether patients had evidence of ICMP versus NICMP, female gender, and wide QRS > $150 \mathrm{~ms}$. Other variables including age, race, body mass index, diabetes mellitus, hypertension, atrial fibrillation, history of $\mathrm{CABG}$, smoking, evidence of LBBB or LBBB duration, medication use, and kidney function did not significantly impact the outcome.

When comparing the NYHA subgroup between patients with ICMP and NICMP, we have noticed that those with ICMP had only modest benefits $(37.8 \%)$, and $51 \%$ had no improvement and $11.2 \%$ actually had worsening in their NYHA functional class. On the other hand, in the 


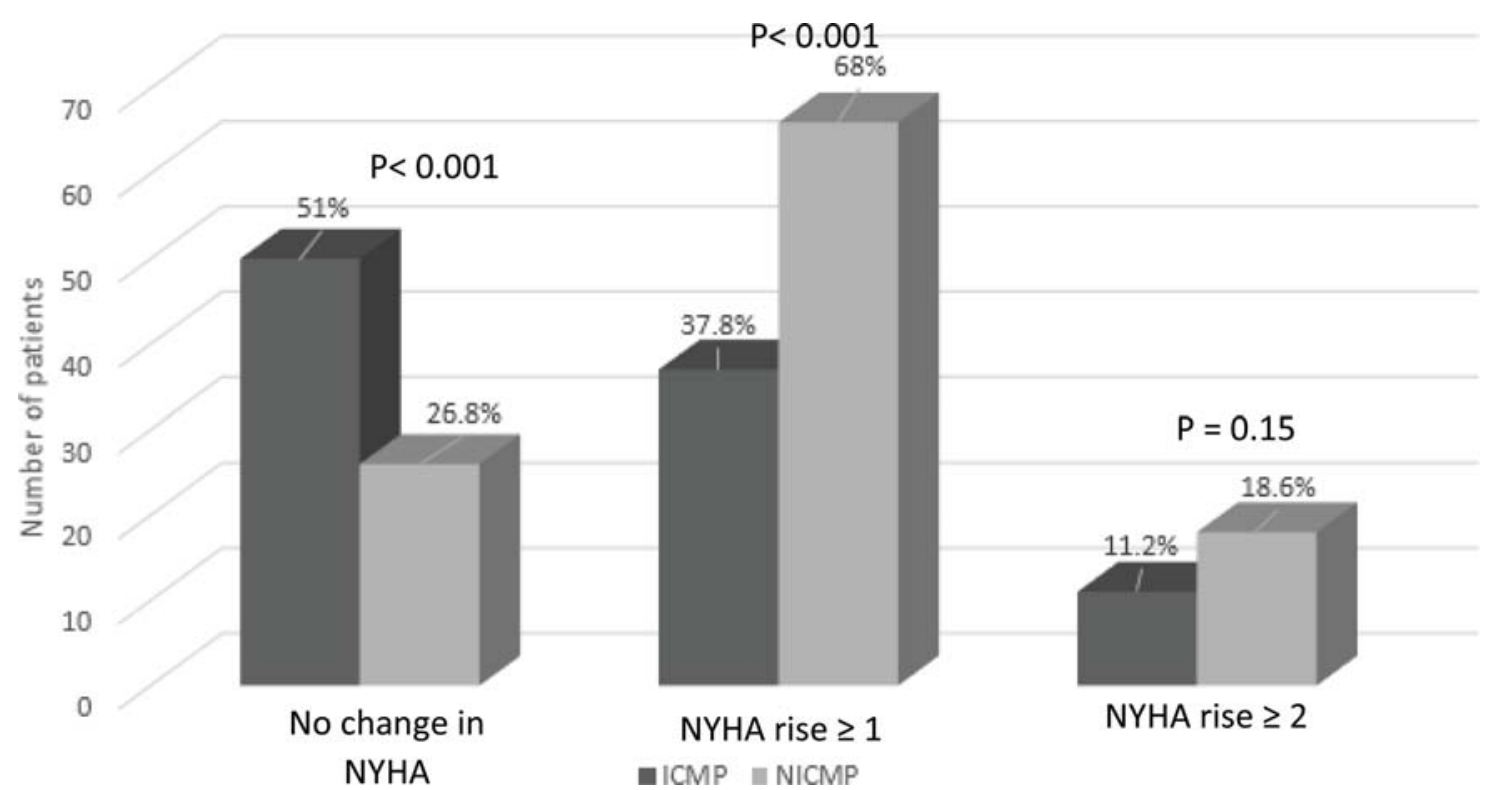

Figure 1: Changes in New York Heart Association category in ICMP and NICMP.

Table 3: Multivariate analysis using logistic regression

\begin{tabular}{lclc}
\hline Variable & Odds ratio & $\mathbf{p}$-Value & $\mathbf{9 5} \%$ confidence interval \\
\hline NICMP & 2.73 & 0.003 & $1.42-5.2$ \\
Female gender & 2.44 & 0.02 & $0.20-0.87$ \\
LBBB & 1.33 & 0.42 & $0.66-2.70$ \\
AFIB & 1.04 & 0.91 & $0.54-2.01$ \\
QRS $>150$ & 2.51 & 0.006 & $1.29-4.86$ \\
\hline
\end{tabular}

AFIB: Atrial Fibrillation; LBBB: left bundle branch block; NICMP: non-ischemic cardiomyopathy.

NICMP group, the NYHA class had improved in nearly two-thirds of the patients $(68 \%)$, did not change in $27 \%$ of the patients, and deteriorated in only $5 \%$ if this subpopulation.

Our study, with its pragmatic design, evaluated CRT outcomes in everyday patients in the real-life setting. The current randomized controlled trials were not specially designed to determine the role of heart failure etiology on the outcomes of CRT. Results from prior observational studies looked into CRT outcomes taking into consideration the etiology of heart failure showed inconsistencies regarding which etiologic subgroup had better outcomes, although the majority demonstrated that NICMP patients exhibited a lower risk for mortality or heart failure hospitalization than ICMP patients.

Whether ischemic etiology really affected the benefits of CRT is not known. In one study, it has been reported that the poor outcomes of ICMP patients were directly related to the large scar burden and fewer viable cardiomyocytes. ${ }^{15}$ Hence, the apparent lower usefulness of CRT in the ICMP subgroup might be related to the disease itself rather than the lower efficacy of CRT treatment.

Limitations to the study include variable follow-up time in NYHA classification and EF assessment, lack of medication adherence appraisal due to the retrospective nature of the study, lack of a control group with no CRT, and unassessed functional aerobic capacity by the 6-minute walk test.

In conclusion, our study shows an improvement in NYHA functional class and EF after CRT therapy in heart failure patients regardless of etiology. However, a more favorable outcome was noticed in the NICMP subgroup. A future randomized control trial, with a control group that specifically addresses this question, and investigates possible etiologies is warranted.

\section{References}

1. Djoussé L, Driver JA, Gaziano JM. Relation between modifiable lifestyle factors and lifetime risk of heart failure. JAMA. 2009;302(4):394-400.

2. Go AS, Mozaffarian D, Roger VL, et al. Heart disease and stroke statistics-2013 update: A report from the American Heart Association. Circulation. 2013;127(1):e6-e245.

3. Heidenreich PA, Trogdon JG, Khavjou OA, et al. Forecasting the future of cardiovascular disease in the united states: A policy statement from the American Heart Association. Circulation. 2011;123(8):933-944.

4. Khand A, Gemmel I, Clark AL, Cleland JG. Is the prognosis of heart failure improving? J Am Coll Cardiol. 2000;36(7): 2284-2286.

5. Trautmann SI, Kloss M, Auricchio A. Cardiac resynchronization therapy. Curr Cardiol Rep. 2002;4(5):371-378.

6. Bristow MR, Saxon LA, Boehmer J, et al. Cardiac-resynchronization therapy with or without an implantable defibrillator in advanced chronic heart failure. $N$ Engl J Med. 2004;350(21):2140-2150.

7. Cleland JG, Daubert JC, Erdmann E, et al. The effect of cardiac resynchronization on morbidity and mortality in heart failure. N Engl J Med. 2005;352(15):1539-1549.

8. Moss AJ, Hall WJ, Cannom DS, et al. Cardiac-resynchronization therapy for the prevention of heart-failure events. $N$ Engl J Med. 2009;361(14):1329-1338. 
9. Tang AS, Wells GA, Talajic M, et al. Cardiac-resynchronization therapy for mild-to-moderate heart failure. $N$ Engl J Med. 2010;363(25):2385-2395.

10. Cazeau S, Leclercq C, Lavergne $T$, et al. Effects of multisite biventricular pacing in patients with heart failure and intraventricular conduction delay. N Engl J Med. 2001;344(12):873-880.

11. Young JB, Abraham WT, Smith AL, et al. Combined cardiac resynchronization and implantable cardioversion defibrillation in advanced chronic heart failure: The MIRACLE ICD Trial. JAMA. 2003;289(20):2685-2694.

12. Abraham WT, Fisher WG, Smith AL, et al. Cardiac resynchronization in chronic heart failure. N Engl J Med. 2002; 346(24):1845-1853.

13. Higgins SL, Hummel JD, Niazi IK, et al. Cardiac resynchronization therapy for the treatment of heart failure in patients with intraventricular conduction delay and malignant ventricular tachyarrhythmias. J Am Coll Cardiol. 2003; 42(8):1454-1459.

14. Tracy CM, Epstein AE, Darbar D, et al. 2012 ACCF/AHA/ HRS focused update of the 2008 guidelines for devicebased therapy of cardiac rhythm abnormalities: A report of the American College of Cardiology Foundation/American Heart Association task force on practice guidelines and the Heart Rhythm Society. Circulation. 2012;126(14): 1784-1800.

15. Adelstein EC, Tanaka H, Soman $P$, et al. Impact of scar burden by single-photon emission computed tomography myocardial perfusion imaging on patient outcomes following cardiac resynchronization therapy. Eur Heart J. 2011;32(1):93-103. 